\title{
WINDS FROM DISKS
}

\author{
J.E.Drew \\ Department of Astrophysics, \\ Keble Road, \\ Oxford $\quad$ OX1 3RH
}

\section{Introduction}

The disks referred to in the title of this paper are specifically those present in cataclysmic variables in which the accreting white dwarf has a relatively weak magnetic field ( $\ll 1 \mathrm{MG}$ ). Such systems are classified either as nova-like variables or as dwarf novae, and are of interest here because they are believed to be novae in quiescence (Ritter and Livio discuss this point elsewhere in this volume).

This review aims to do two things: $i)$ to summarise what has been learned about the winds associated with non-magnetic cataclysmic variables both from observation and from numerical modelling, and ii) to outline ideas about the nature of the mass loss mechanism. By contrast with the certainty that nova outflows are the consequence of thermonuclear runaway, it shall be seen that the fundamental cause of mass loss from cataclysmic variables remains obscure. An earlier review of this subject is by Cordova and Howarth (1986). Also of interest are some sections of the monograph on dwarf novae and nova-like variables by LaDous (1989).

\section{Wind characteristics}

\subsection{Deductions from observations}

The existence of winds from the disks in nova-like variables and dwarf novae is a discovery made by the International Ultraviolet Explorer satellite (IUE). Optical evidence of mass loss from these systems is, at best, subtle (see, for example, the optical study of SW Sex by Honeycutt et al., 1986) and has not yet provided any useful insights into its nature. Hence, at present, a discussion of what is known from observation about cataclysmic variable (CV) winds need only refer to the body of data gathered during the 11 years or so of IUE's lifetime.

The primary evidence of outflow from CVs is the presence in their spectra of broad, blueshifted absorption in the profiles of the higher ionisation ultraviolet resonance lines. The transitions that are always affected are CIV $\lambda 1549$, SiIV $\lambda 1397$ and NV $\lambda 1240$. The total breadth of the blueshifted absorption corresponds to. velocities in the range $3000-5000 \mathrm{~km} \mathrm{~s}^{-1}$. This phenomenon is associated only with non-eclipsing nova-like 


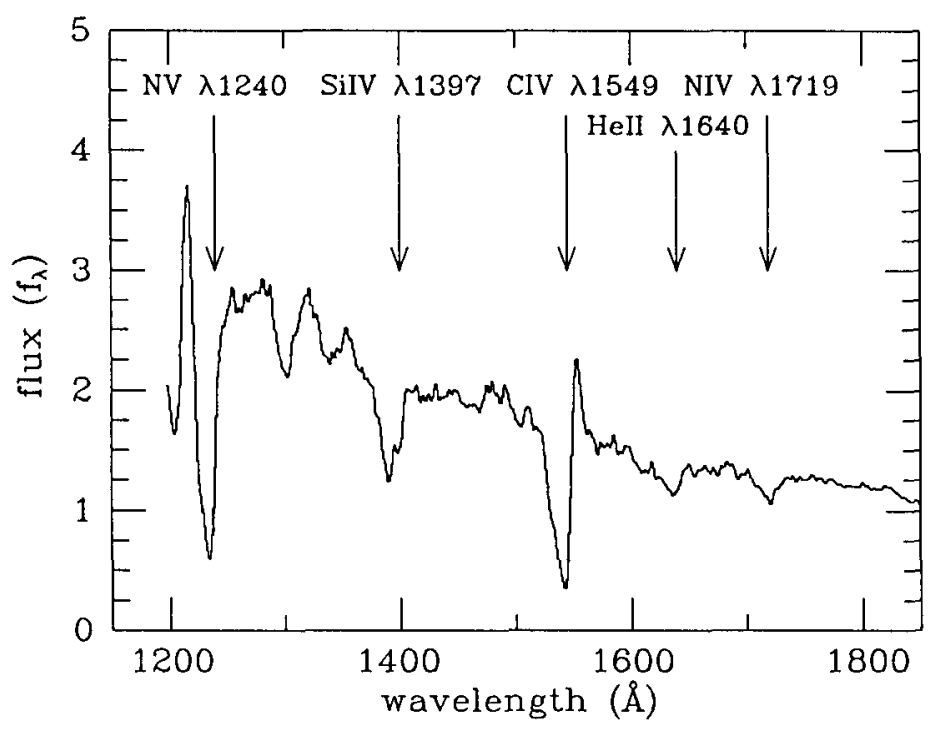

Figure 1: The average of three consecutive IUE spectra of the nova-like variable, RW Sex (from image numbers SWP 16269-71). The blueshifted absorption is particularly deep in this object and, unusually, is probably present in HeII $\lambda 1640$ and NIV $\lambda 1719$.

variables and dwarf novae in outburst. In both types of system the mass accretion rate is high $\left(\dot{M}_{\text {acc }} \gtrsim 10^{-9} \mathrm{M}_{\odot} \mathrm{yr}^{-1}\right)$. An example of the short wavelength spectrum of one such system, RW Sex, is shown in Figure 1. Blueshifted absorption is not observed in either the spectra of eclipsing systems or in quiescent dwarf novae whose mass accretion rates are much lower $\left(\dot{M}_{\text {acc }} \lesssim 10^{-10} \mathrm{M}_{\odot} \mathrm{yr}^{-1}\right)$.

The simple fact that velocities of up to $\sim 5000 \mathrm{~km} \mathrm{~s}^{-1}$ are attained in these winds was quickly interpreted as a likely indication of mass loss originating from the vicinity of the white dwarf (e.g., Cordova and Mason, 1982). This is because the escape speed from the surface of a white dwarf is of this order $\left(v_{\text {esc }} \simeq 5000\left(M / M_{\odot}\right)^{0.5}\left(R / 10^{9} \mathrm{~cm}\right)^{-0.5}\right.$ $\mathrm{km} \mathrm{s}^{-1}$ ) and is thus the magnitude of expansion velocity expected of an outflow initiated in its neighbourhood. In contrast, there is no a priori reason why mass loss from the outer parts of a CV accretion disk should be accelerated up to speeds many times the local escape speed. This is partly why numerical modelling has concentrated on outflow geometries in which the mass loss originates from close to the accreting white dwarf.

The form of the wind-dominated ultraviolet resonance line profiles changes radically as the system's inclination to the line of sight rises to the point that continuum eclipses begin to be observed. Broad blueshifted absorption is no longer apparent in the CIV, NV and SiIV resonance line profiles. Instead, these transitions are observed in emission, their profiles being velocity-broadened $\left(\Delta v_{F W Z I} \lesssim 5000 \mathrm{~km} \mathrm{~s}^{-1}\right)$ and roughly symmetric. Examples of such spectra are shown in Figure 2. Since all eclipsing non-magnetic CVs are too faint to observe at high spectral resolution with IUE, it is not yet possible to 
UXUMa 1981 Nov 8

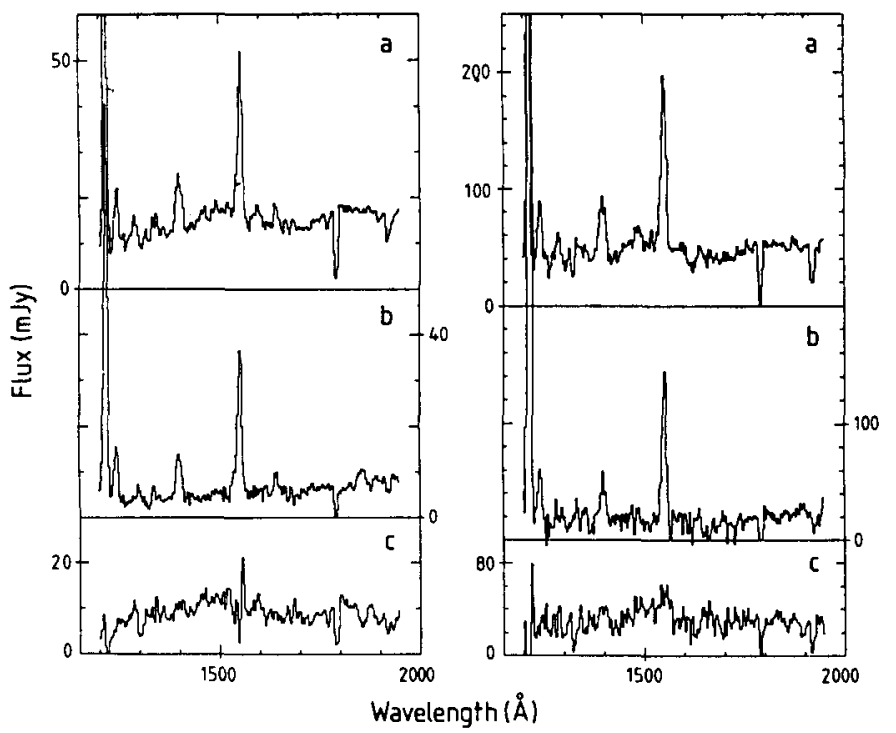

Figure 2: Average UV spectra of the nova-like variables, UX UMa and RW Tri, (a) away from eclipse and (b) during eclipse. In (c) the difference (a-b) is shown. That the emission lines do not show up in the difference spectra is evidence of their shallow eclipse. (Figure from Drew and Verbunt, 1985)

firmly rule out the presence of weak blueshifted absorption. However, it is clear that the line-forming region has to be bipolar in some respect in order to explain so pronounced a dependence on inclination angle. Mauche and Raymond (1987) have argued that the bipolarity inherent in the radiation field (aided by limb-darkening) is sufficient to produce this effect. Drew (1987) considered that the limb-darkening needed was too extreme, and so preferred to explain the absence of blueshifted absorption in eclipsing systems in terms of a modestly bipolar outflow.

A most striking property of the resonance line emission observed in eclipsing systems is that it suffers little eclipsing even when the continuum suffers deep eclipses (King et al., 1983; Cordova and Mason, 1985; Naylor et al., 1988). The lower panels in Figure 2 illustrate this point very clearly. The usual pattern is that CIV $\lambda 1549$ eclipses barely at all, with the eclipses of NV $\lambda 1240$ being somewhat deeper and those of SiIV $\lambda 1397$, the deepest. The clear implication of this behaviour is that the sizes of the line-forming regions are of the same order as that of the occulting secondary star. In such cases, $\dot{M}<10^{-10} \mathrm{M}_{\odot} \mathrm{yr}^{-1}$ is probably ruled out (Drew, 1987).

Enough IUE observations have been made of nova-like variables and outburst dwarf novae to reveal two types of regular variability in the wind-formed line profiles. These are a dependence on outburst phase (for dwarf novae only, of course), and an orbital phase dependence. The typical pattern of variation through a dwarf nova outburst is for the blueshifted absorption (in non-eclipsing systems) to be most marked at the peak of the outburst, and then to diminish as the overall luminosity of the system begins to decay 


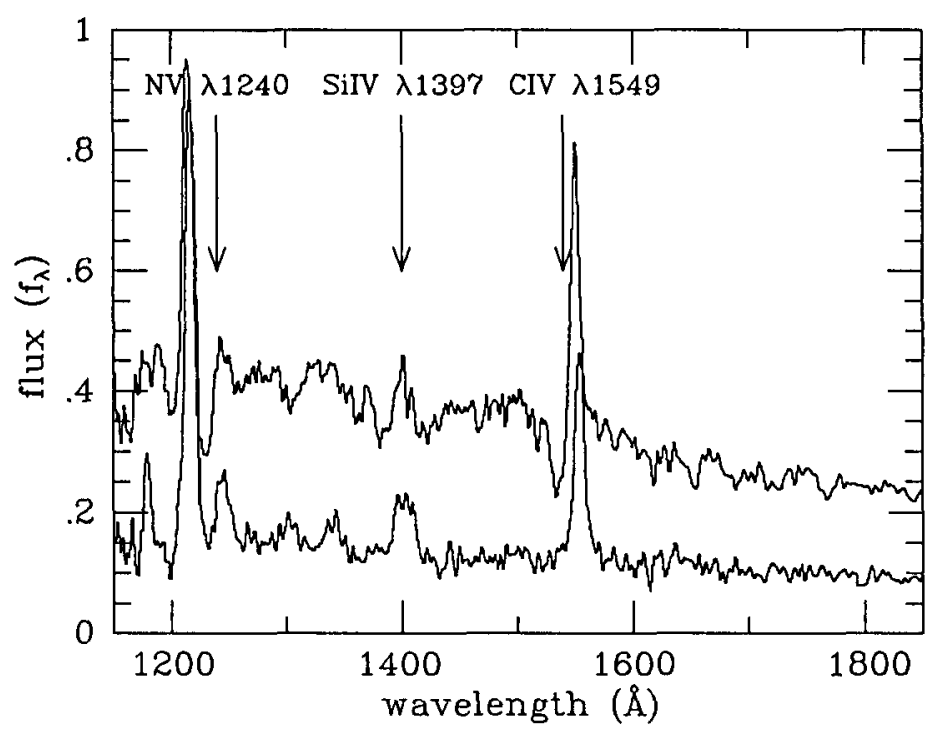

Figure 3: Two outburst IUE spectra of the dwarf nova, SU UMa, obtained a day apart (image numbers SWP 34823 and 34832). The later spectrum is the lower of the two. The same flux scale applies to both spectra.

(Verbunt et al., 1984; Drew and Verbunt, 1988). Recently obtained outburst spectra of the dwarf nova, SU UMa, provide a particularly clear example of this behaviour (Woods et al., 1989). Two of these spectra, obtained a day apart and at the same orbital phase, are shown in Figure 3. In the later spectrum, the continuum level has fallen by a little over a factor of two, and the absorption associated with the resonance lines has disappeared, leaving roughly symmetric emission profiles similar to those observed during quiescence. In the earlier spectrum, taken at about maximum light, blueshifted absorption is very clearly present. The most plausible interpretation of this behaviour is that the mass loss rate is strongly dependent on the system luminosity. It follows from this that mass loss is negligible or absent in the quiescent state. Unfortunately, this view is not easily tested because quiescent dwarf novae lack the optically thick, continuum-emitting disks against which outflow can be detected.

The dependence of wind-formed resonance line profiles upon orbital phase varies from being slight or negligible in most systems to extreme in one case. The extreme example, the dwarf nova $\mathrm{YZ} \mathrm{Cnc}$, was uncovered when it was observed continuously over several orbital cycles (Drew and Verbunt, 1988). The behaviour of its UV spectrum is illustrated in Figure 4. Two spectra obtained half an hour apart are shown: in the first the blueshifted absorption is weak, while in the second (about 0.25 of an orbital cycle later) it is very prominent. During the rest of the orbital cycle, the absorption gradually diminishes again. Observations of this system are now sufficiently extensive to indicate that line profile variations on the orbital timescale are present in all outbursts. 

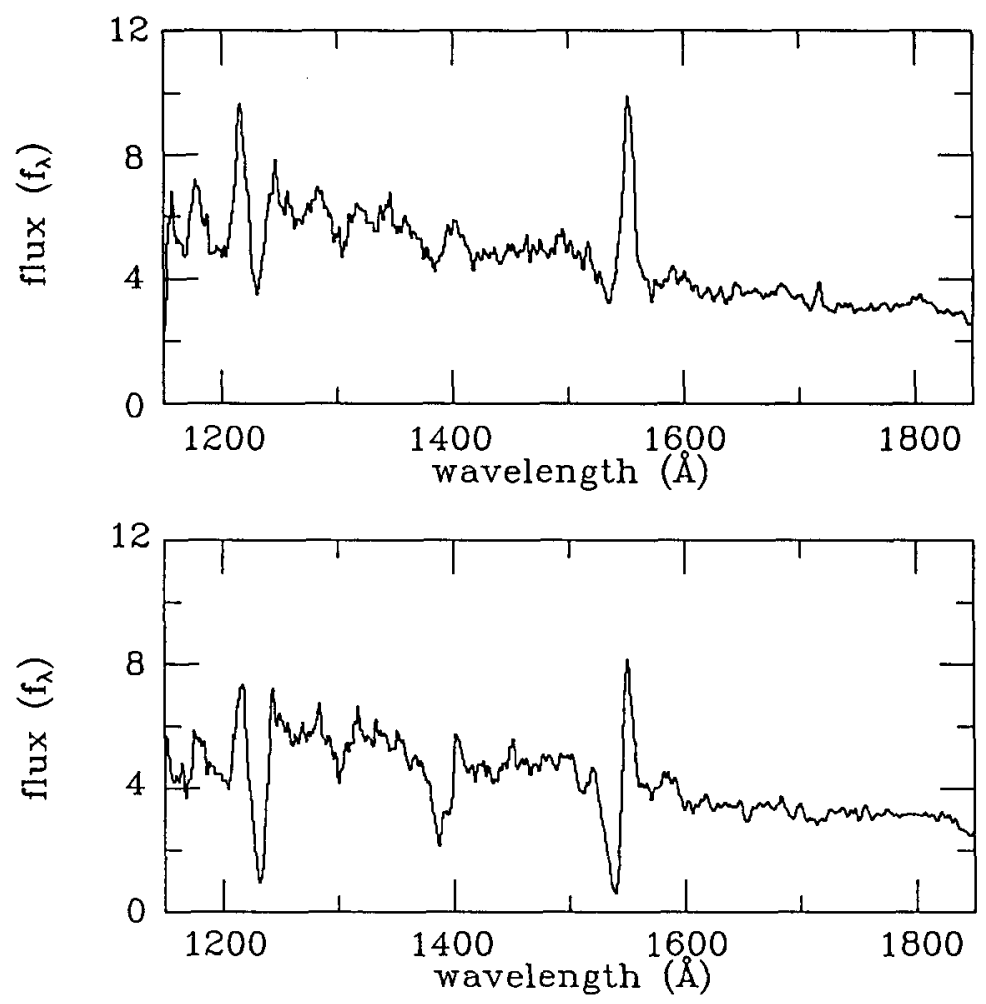

Figure 4: Two outburst spectra of the dwarf nova, YZ Cnc, obtained in the same IUE image (SWP 30437) half an hour apart.

Of the half-dozen systems that have been observed in a way that reveals orbital phase variations, phase-dependent resonance line profiles have been found in three ( $\mathrm{YZ} \mathrm{Cnc,}$ SU UMa (Woods et al., 1989), Z Cam (Szkody and Mateo, 1986)). The fact that detectable orbital phase dependence is probably not abnormal suggests that there may be a significant departure from axial symmetry in the disk/wind geometry. Whether the asymmetry exists in the wind or in the disk is entirely unclear.

\subsection{Results of numerical modelling}

Two kinds of numerical modelling have been undertaken. Firstly, ionisation models have been constructed for a wind illuminated by a disk and boundary layer continuum radiation field (Drew and Verbunt, 1985; Kallman and Jensen, 1985; Mauche and Raymond, 1987). The main aim of this work was to fix the scale of the mass loss rates involved. Secondly, Drew (1987) and Mauche and Raymond (1987) have synthesised model UV resonance line profiles with a view to setting constraints on the wind geometry and velocity law. All these studies share the same fundamental assumptions: 
that the outflow originates from close to the white dwarf surface and that it is radial. These assumptions have been applied in the spirit that the simplest geometry must be explored first. In addition, Drew and Verbunt (1985) and Mauche and Raymond (1987) loosely justify this geometry on the grounds that radiation pressure due to the inner disk and boundary layer is very likely to be an important factor in determining the wind dynamics.

One of the main results to have emerged from the line profile synthesis carried out by Drew (1987) and Mauche and Raymond (1987) concerns the wind velocity law (the dependence of expansion velocity on radius). Both studies find that the observed blueshifted absorption profiles cannot be reproduced using the steep velocity laws that are believed to be appropriate to the radiation-pressure-driven winds of hot stars. The difficulty with such sharply accelerated winds (in which $v \rightarrow v_{\infty}$ for $r \lesssim 3 R$, where $R$ is the stellar radius), is that for suitable concentrations of the resonantly scattering ions, the maximum absorption should be achieved at the shortest wavelengths in the resonance line profiles, rather than at close to line centre as observed (see Figures 1-4). If the outflow is assumed to be accelerated much more slowly, such that $v \rightarrow v_{\infty}$ for $r \sim R_{\text {disk }}$, then the synthesised line profiles do take on an appropriate form. Mauche and Raymond (1987) note that this follows directly from the fact that the ultraviolet continuum photons scattered in the wind are produced in the accretion disk out to radii as large as $10 R$ or more.

By comparing synthesised and observed line profiles, it is possible to set limits on $\dot{M}_{q}$ where $q$ is the fractional abundance of the scattering ion. Drew (1987) estimated $\dot{M}_{q_{C^{3}}} \gtrsim 10^{-10} \mathrm{M}_{\odot} \mathrm{yr}^{-1}$ from eclipse observations of the nova-like variables, UX UMa and RW Tri. Mauche and Raymond (1987) derived $\dot{M}_{q_{C^{3}}} \sim 10^{-11} \mathrm{M}_{\odot} \mathrm{yr}^{-1}$ for the lower inclination dwarf nova, HL CMa. However, to separate the ionisation fraction from the mass loss rate, a model of the wind ionisation structure is essential.

Without a doubt, the most telling ionisation calculation performed to date is due to Mauche and Raymond (1987). The earlier work by Drew and Verbunt (1985) stopped short by leaving out of consideration mass loss rates in excess of $\dot{M}_{\text {acc }} / 20$, while Kallman and Jensen (1985) abstracted the problem in such a way that their conclusion concerning the required $\dot{M}$ s did not clinch the case. Mauche and Raymond's most important and, indeed, most disturbing finding was that $\dot{M} / \dot{M}_{\text {acc }} \gtrsim 1 / 3$ is required. This allows only a narrow range of acceptable of mass loss rates since $\dot{M} / \dot{M}_{a c c}>1$ is impossible if the wind's energy source is accretion!

The reason for the high $\dot{M}$ estimate is that the fiercely-ionising boundary layer radiation in the $\mathrm{He}^{+}$Lyman continuum has to be blotted out by the inner wind before the concentrations of $C^{3+}$ can rise to the level needed to produce the observed UV line profiles. Mauche and Raymond considered the possibility that CV winds might be inhomogeneous due to shocking in the same way that the radiation-driven winds of hot stars are thought to be (Castor, 1987). This achieved a saving of about a factor of 3 in the required mass loss rate (otherwise $\dot{M} \sim \dot{M}_{\text {acc }}$ would have been obtained). However, if it is also assumed that the outflow is enhanced over the white dwarf poles (i.e. it is bipolar), the same radial density profile can be achieved in the line-forming region for still lower mass loss rates. In this way, Mauche and Raymond's lower limit on the ratio $\dot{M} / \dot{M}_{\text {acc }}$ can be lowered by another factor of a few. 


\section{Implications for the mass loss mechanism}

$A b$ initio modelling of the dynamics of disk winds in cataclysmic variables is greatly hampered by two outstanding problems in accretion theory: these are the nature of the vertical disk structure (which is bound up with the failure to identify the source of viscosity) and the character of the boundary layer region.

In order to construct a radiation-pressure driven wind model, using both the boundary layer and inner disk radiation, it is necessary to define the geometry of the boundary layer radiation field and also its energy dependence. Both are unknown, but radiation temperatures somewhat in excess of $10^{5} \mathrm{~K}$ are expected (Pringle and Savonije, 1979; Patterson and Raymond, 1985). A thorough exploration of this class of model has yet to be published (although it seems that Ballegooijen and Raymond have a paper in preparation). An alternative scenario that has been discussed in the literature is a centrifugal MHD disk wind model of the type initially applied to protostellar sources (Pudritz and Norman, 1986; Cannizzo and Pudritz, 1988). A lack of initial constraints is again a problem because neither the disk's magnetic field, nor the way matter might be injected into an outflow can be determined without any real understanding of the disk's vertical structure. In view of these problems in setting up the dynamical models, it is useful to make some general remarks about their plausibility in the light of the available observations. The upshot is that both have serious defects.

The most serious difficulty faced by the radiation-driven wind model is the high mass loss rates implied by the need to produce observable quantities of ions such as $C^{3+}$. It is easily shown that the 'singe-scattering' momentum limit, $L /\left(c \dot{M} v_{\infty}\right)$ is exceeded if $\dot{M} / \dot{M}_{\text {acc }} \gtrsim 0.01$ (Drew and Verbunt, 1985). Mauche and Raymond's (1987) results have shown that this limit is exceeded by about an order of magnitude. This cannot be the case. One possible resolution of this problem is that the wind is somehow shielded from the boundary layer radiation, thus allowing the appropriate ion densities to exist in a less massive outflow.

Another rather more nebulous difficulty for the radiation-driven wind model is that the slow acceleration deduced from the observed absorption profiles in low-inclination systems may well be hard to achieve. This is because the radiation-pressure force can be expected to decline in proportion to the dilution of the radiation field. While it is true that the extended nature of the EUV continuum source (boundary layer and inner disk) results in more gradual dilution of the radiation field than in the case of a single star, it may not be gradual enough. Kallman (1987) has considered this issue and estimates that terminal velocity would be approached within about $10 R$ dwarf radii (instead of 3 , as for a star). It is extremely unlikely that, in addition, changing wind ionisation can assist in accelerating the outflow by causing an outward increase in wind opacity.

The main problem with the centrifugal MHD wind model, as applied by Cannizzo and Pudritz (1988) to cataclysmic variables, is that the preferred site of mass loss is the outer part of the accretion disk. The likely consequence of this is that the inner UVemitting portion of the disk will be most effectively occulted by the outflow when the disk is highly inclined to the line of sight. Hence, the reverse to the observed pattern of prominent blueshifted absorption in low-inclination systems and little or no absorption in eclipsing systems is implied. This is surely fatal to the model in its present form. If, 
instead, the inner disk can be the source of the outflow, then the model may have a future.

\section{Postscript}

Clearly, a satisfactory mechanism for driving winds from accretion disks in cataclysmic variables has yet to be worked out. It seems that the inner disk is the most promising site for the initiation of the outflow. This said, much work is ahead in determining the dynamical roles played by radiation pressure and/or magnetic fields (due either to the disk itself or the accreting white dwarf). It will be important to include in the dynamics a proper account of the effect of the rotational motion of gas entering the flow. However, in the near future, it is likely that we will learn more about these winds by piecing together new observational constraints rather than by building complex theories from uncertain premises. Indeed, it is to be hoped that soon, ROSAT, will give us some desperately need insights, particularly into that troublesome boundary layer!

\section{References}

Cannizzo,J.K., Pudritz, R.E., 1988, Astrophys. J., 327, 840.

Castor, J.I., 1987, in 'Instabilities in Luminous Early-Type Stars', eds. H.J.G.L.M. Lamers and C.W.H. de Loore, Reidel, pp 159-170.

Cordova, F.A., Howarth, I.D., 1986, in 'Exploring the Universe with the IUE Satellite', eds. Y. Kondo et al., Reidel pp 395-426.

Cordova, F.A., Mason, K.O., 1982, Astrophys. J., 260, 716.

Cordova, F.A., Mason, K.O., 1985, Astrophys. J., 290, 671.

Drew, J.E., 1987, Mon. Not. R. ast. Soc., 224, 595.

Drew, J.E., Verbunt, F., 1985, Mon. Not. R. ast. Soc., 213, 191.

Drew, J.E., Verbunt, F., 1988, Mon. Not. R. ast. Soc., 234, 341.

Honneycutt, R.K., Schlegel, E.M., Kaitchuk, R.H., 1986, Astrophys. J., 302, 388.

Kallman, T.R., 1987, in proc. IAU/COSPAR conference, 'Accretion onto Compact Objects', held in Sofia, Bulgaria, in press.

Kallman, T.R., Jensen, K.A., 1985, Astrophys. J., 299, 277.

King, A.R., Frank, J., Jameson, R.F., Sherrington, M.R., 1983, Mon. Not. R. ast. Soc., 203, 677 .

LaDous, C., 1989, in 'Cataclysmic Variables and Related Objects', ed. M.Hack, NASA/CNRS Monograph Series on Non-Thermal Phenomena in Stellar Atmospheres.

Mauche, C.W., Raymond, J.C., 1987, Astrophys. J., 323, 690.

Naylor, T., et al., 1988, Mon. Not. R. ast. Soc., 231, 237.

Patterson, J., Raymond, J.C., 1985, Astrophys. J., 292, 505.

Pringle, J.E., Savonije, G.J., 1979, Mon. Not. R. ast. Soc., 210, 197.

Pudritz, R.E., Norman, C.A., 1986, Astrophys. J., 301, 571.

Szkody, P., Mateo, M., 1986, Astrophys. J., 301, 286.

Verbunt, F., et al., 1984, Mon. Not. R. ast. Soc., 210, 197.

Woods, J.A., Drew, J.E., Verbunt, F., 1989, in preparation. 